\title{
Actividad de cobre sobre cocáceas grampositivas multi-resistentes a los antibióticos aisladas en hospitales chilenos
}

\author{
Tomás Kappes, Mariana Domínguez, Helia Bello-Toledo, Sergio Mella Montecinos, \\ Gisella Riedel Molina y Gerardo González-Rocha
}

\section{Copper activity against multiresistant Gram-positive cocci isolated in Chilean hospitals}

Background: Nosocomial infections caused by multiresistant Gram-positive cocci are a serious problem for public health systems worldwide. The use of copper surfaces in hospital environments has proven to be an effective alternative for the control of various microorganisms, including multiresistant nosocomial pathogens. Aim: To determine the association between antibiotic multiresistance and higher levels of copper tolerance in Gram-positive cocci isolated from Chilean hospitals, which might confer a selective advantage in environments with copper. Methods: The ionic copper tolerance levels were evaluated using the Mueller Hinton agar dilution method, in $S$. aureus and Enterococcus spp. strains with different levels of susceptibility to clinically relevant antibiotics. Results: A statistically significant association between higher levels of tolerance to copper ion and multi-resistance to antibiotics in Enterococcus spp. was observed.

Key words: Copper tolerance, antibiotic resistance, nosocomial infections.

Palabras clave: Tolerancia a cobre, resistencia antimicrobiana, infecciones nosocomiales.

\section{Introducción}

A ctualmente las infecciones asociadas a la atención en salud (IAAS), producidas por bacterias nosocomiales multi-resistentes a los antimicrobianos, constituyen un grave problema mundial de salud pública, al que se asocia un aumento de las tasas de morbimortalidad, así como del tiempo de hospitalización de los pacientes que las adquieren ${ }^{1}$, generando un considerable incremento en los costos del tratamiento de estas enfermedades infecciosas. Dentro de los agentes etiológicos causantes de estas infecciones, destacan ciertas especies cocáceas grampositivas como Staphylococcus aureus y Enterococcus spp. por su prevalencia, principalmente en infecciones de herida operatoria y bacteriemias ${ }^{2}$. En estas especies, los fenotipos de mayor relevancia clínica, por la dificultad de tratar las infecciones que causan, son las cepas de $S$. aureus resistentes a meticilina (SARM) y de Enterococcus spp. resistentes a vancomicina (ERV) ${ }^{3,4}$. Debido a las limitadas alternativas terapéuticas, producto del constante aumento de los niveles de resistencia a antimicrobianos observados en estos patógenos, se ha postulado el uso de cobre como antimicrobiano de superficie en hospitales como una alternativa en el control de los patógenos nosocomiales en estos ambientes ${ }^{5,6}$.

Las propiedades antimicrobianas de las aleaciones de cobre han sido comprobadas en diversos estudios sobre diferentes patógenos ${ }^{7,8} ;$ no obstante, se ha descrito que la multi-resistencia a antimicrobianos en ciertas especies bacterianas podría asociarse con la tolerancia a cobre iónico $^{9-11}$, confiriéndoles ventajas selectivas potenciales en estas superficies ${ }^{12}$. Debido a esto, cepas multi-resistentes a antimicrobianos que presenten mayores niveles de tolerancia a cobre, podrían sobrevivir más tiempo en presencia de este metal y de este modo ser seleccionadas en ambientes donde se utilice el cobre como antimicrobiano de superficie.

En investigaciones realizadas anteriormente ${ }^{13}$, se determinó la existencia de una asociación estadísticamente significativa entre perfiles extendidos de resistencia a antimicrobianos y la tolerancia a cobre en bacterias gramnegativas aisladas en hospitales chilenos; en este estudio se investigó la actividad de cobre iónico $\left(\mathrm{CuSO}_{4}\right)$ sobre cepas de $S$. aureus resistentes y sensibles a meticilina (SASM) así como de Enterococcus spp. resistentes y sensibles a vancomicina (ESV), para establecer relaciones entre la resistencia a antimicrobianos y la tolerancia a cobre.

\section{Material y Método}

\section{Cepas bacterianas}

Se analizaron 202 cepas de bacterias cocáceas grampositivas aisladas en hospitales chilenos, entre los años 1997 y 2011. Estas cepas pertenecen a la colección del
Universidad de Concepción, Concepción, Chile. Facultad de Ciencias Biológicas Departamento de Microbiología Laboratorio de Investigación en Agentes Antibacterianos (TK, MD, HBT, GGR).

Facultad de Medicina,

Departamento de Medicina Interna (SMM, GRM).

Hospital Regional “Dr. Guillermo Grant Benavente", Concepción, Chile.

Unidad de Infectología (SMM, GRM)

No existen conflictos de interés Fuente de financiamiento: Facultad de Ciencias Biológicas. Universidad de Concepción.

Recibido: 29 de diciembre de 2015 Aceptado: 27 de agosto de 2016

Correspondencia a: Gerardo González-Rocha ggonzal@udec.cl 
Laboratorio de Investigación en Agentes Antibacterianos, Departamento de Microbiología, Facultad de Ciencias Biológicas de la Universidad de Concepción y a la colección de cepas del Instituto de Salud Pública de Chile. Se incluyeron 102 cepas de $S$. aureus (52 SARM y 50 SASM) y 100 cepas de Enterococcus spp. [50 ERV (E. faecium) y $50 \mathrm{ESV}$ (25 E. faecium y 25 E. faecalis)]. Las cepas $S$. aureus ATCC 29213 y $S$. aureus ATCC 25923, fueron utilizadas como cepas controles de calidad en los estudios de susceptibilidad.

\section{Estudio de susceptibilidad a agentes antibacterianos}

El estudio de susceptibilidad a agentes antibacterianos se realizó mediante el método de difusión en agar Mueller-Hinton (Oxoid), siguiendo las recomendaciones y puntos de corte propuestos por el Clinical and Laboratory Standards Institute (CLSI) ${ }^{14}$, con excepción de la determinación de la actividad de vancomicina sobre $S$. aureus, que fue investigada mediante el método de dilución seriada en agar Mueller-Hinton siguiendo las metodologías propuestas por el CLSI ${ }^{15}$. Los antimicrobianos y la potencia de los discos utilizados fueron: ampicilina (AMP, $10 \mu \mathrm{g}$ ), cefoxitina (FOX, $30 \mu \mathrm{g}$ ), gentamicina (GEN, $120 \mu \mathrm{g}$ ), estreptomicina (STR, $300 \mu \mathrm{g}$ ), tetraciclina (TET, $30 \mu \mathrm{g}$ ), cotrimoxazol (SXT, 1,25/23,75 $\mu \mathrm{g}$ ), cloranfenicol (CHL,

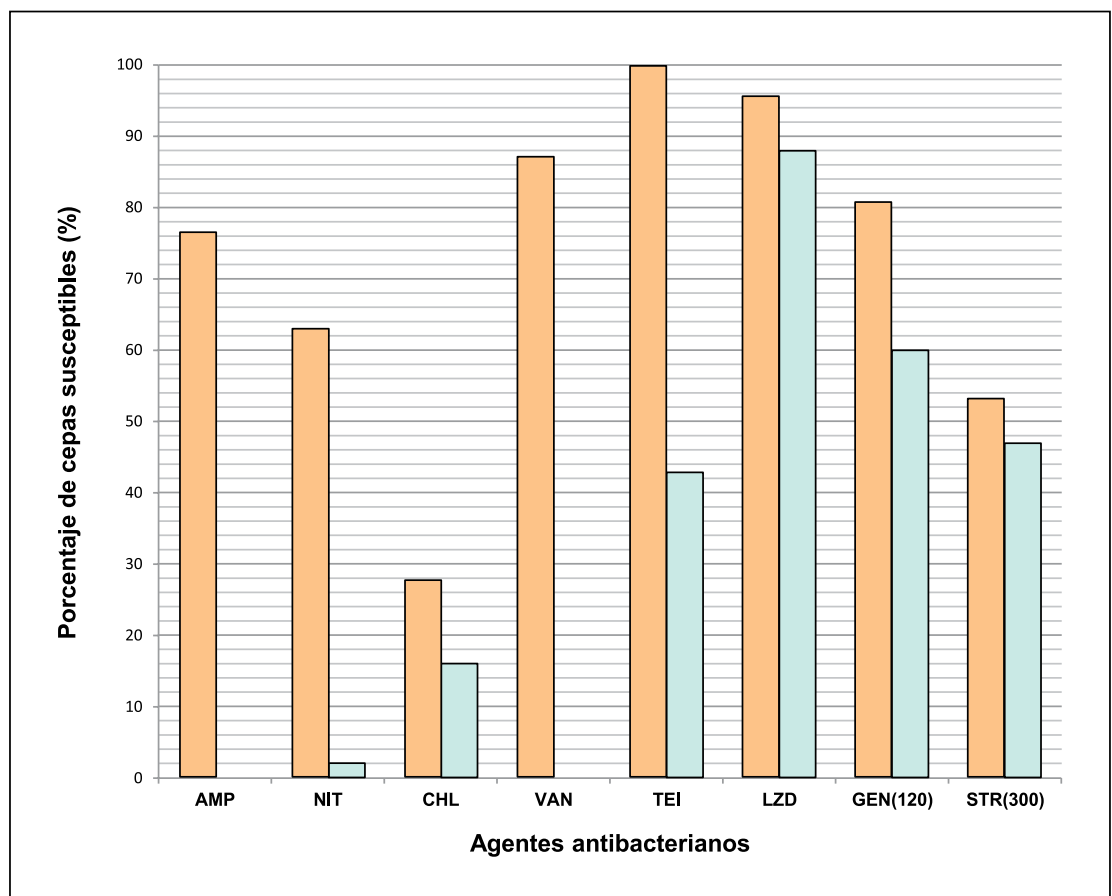

Figura 1. Actividad de agentes antibacterianos de relevancia clínica sobre cepas de Enterococcus spp. sensibles (barras naranja) y resistentes (barras celeste) a VAN. AMP: ampicilina, NIT: nitrofurantoína, CHL: cloranfenicol, VAN: vancomicina, TEl: teicoplanina, LZD: linezolid, GEN(120): gentamicina, STR(300): estreptomicina.
$30 \mu \mathrm{g})$, rifampicina (RIF, $5 \mu \mathrm{g}$ ), vancomicina (VAN, 30 $\mu \mathrm{g}$ ), teicoplanina (TEI, $30 \mu \mathrm{g}$ ), eritromicina (ERY, 15 $\mu \mathrm{g})$, clindamicina (CLI, $2 \mu \mathrm{g})$, linezolid (LZD, $30 \mu \mathrm{g}$ ) y nitrofurantoína (NIT, $300 \mu \mathrm{g})$. En las cepas de Enterococcus spp. se evaluó la actividad de AMP, GEN, STR, CHL, VAN, TEI, LZD y NIT mientras que en $S$. aureus se evaluó FOX, TET, SXT, RIF, VAN, ERY, CLI y LZD.

\section{Actividad antibacteriana de $\mathrm{CuSO}_{4}$}

Se determinó la CIM de cobre mediante ensayos de dilución seriada en placas de agar Mueller Hinton ${ }^{14}$ suplementadas con $\mathrm{CuSO}_{4}$, en un rango de 1 a $16 \mathrm{mM}$. Se utilizó un inóculo final de $10^{4} \mathrm{ufc} / \mathrm{mL}$ por cepa y se incubó a $37^{\circ} \mathrm{C}$ por $48 \mathrm{~h}$.

\section{Análisis estadístico}

Se analizó la significancia estadística de las diferencias de las medias geométricas de los grados de tolerancia a cobre entre los distintos grupos de cepas de las especies estudiadas (cepas resistentes o sensibles a vancomicina o meticilina en Enterococcus spp. y S. aureus, respectivamente). Estas metodologías fueron realizadas siguiendo los procedimientos y parámetros previamente descritos ${ }^{13}$.

\section{Resultados}

Las cepas ERV presentaron menor susceptibilidad a los antimicrobianos evaluados respecto de sus pares ESV (Figura 1). Se observó resistencia intermedia a VAN en $12,8 \%$ de las cepas ESV; la totalidad de las cepas ESV fueron sensibles a TEI, mientras que sólo 42,9\% de las cepas ERV fue sensible a este antimicrobiano. Ambos grupos de cepas presentaron alta susceptibilidad a LZD alcanzando $96 \%$ en ESV y $88 \%$ en ERV. En relación a los aminoglucósidos evaluados, ambos grupos de cepas presentaron mayor frecuencia de cepas sensibles a GEN que a STR. No se observó cepas ERV sensibles a AMP, mientras que la susceptibilidad a este antimicrobiano en cepas de ESV fue de $76 \%$.

Para $S$. aureus se observó que todas las cepas fueron sensibles a LDZ y VAN (Figura 2); además, sólo 3,8\% de las cepas SARM fue resistente a RIF y SXT, sin detectar cepas resistentes a estos antimicrobianos en las cepas SASM. Entre las cepas SASM se observó mayor frecuencia de susceptibilidad a CLI $(84,0 \%)$ y ERY $(56,0 \%)$ que sus pares resistentes a meticilina, cuyas susceptibilidades a CLI y ERY fueron de 51,9 y 23,1\%, respectivamente.

Por su parte, al comparar la media geométrica de la $\mathrm{CIM}$ de $\mathrm{CuSO}_{4}\left(\mathrm{MG}_{\mathrm{CIM}}\right)$ de los grupos de cepas analizadas, se observó que las cepas de ERV presentan niveles de tolerancia a cobre iónico mayores que sus contrapartes sensibles a VAN (Tabla 1). Estas diferencias son estadísticamente significativas con valores de $\mathrm{p}<0,001$ (Tabla 1). 


\begin{tabular}{|c|c|c|c|c|c|c|c|}
\hline Especie bacteriana & Fenotipo de resistencia & n cepas & Rango CIM* & $\mathrm{CIM}_{50}$ & $\mathrm{CIM}_{90}$ & $\mathrm{MG}_{\mathrm{CIM}^{\dagger}}^{\dagger}$ & Valor $p$ \\
\hline \multirow[t]{2}{*}{ Enterococcus spp. } & VAN $^{R}$ & 50 & 2 a 6 & 4 & 6 & 4,63 & \multirow{2}{*}{$<0,001^{t+}$} \\
\hline & VAN $^{5}$ & 50 & 1 a 6 & 2 & 4 & 2,74 & \\
\hline \multirow[t]{2}{*}{ Staphylococcus aureus } & $\mathrm{MET}^{\mathrm{R}}$ & 52 & 1 a 6 & 4 & 6 & 3,55 & \multirow{2}{*}{0,042} \\
\hline & $\mathrm{MET}^{\mathrm{S}}$ & 50 & 1 a 6 & 2 & 6 & 2,76 & \\
\hline
\end{tabular}

En $S$. aureus se observó que las cepas SARM presentan mayores $\mathrm{MG}_{\mathrm{CIM}}$ que los SASM (Tabla 1); sin embargo, esta diferencia no alcanza significancia estadística (Tabla 1). Se observó que en cepas de ERV y SARM la $\mathrm{CIM}_{50}$ de $\mathrm{CuSO}_{4}$ fue de $4 \mathrm{mM}$, pero menor para las cepas sensibles (2 $\mathrm{mM})$.

\section{Discusión}

Las diferencias observadas entre las $\mathrm{MG}_{\mathrm{CIM}}$ de $\mathrm{CuSO}_{4}$ entre los grupos de cepas ERV y ESV permiten establecer una asociación estadísticamente significativa $(\mathrm{p}<0,001)$ entre la resistencia a VAN y el mayor nivel de tolerancia a cobre en cepas de Enterococcus spp. aisladas en centros de salud de Chile (Figura 1). El fenómeno de asociación entre la resistencia a antimicrobianos glicopeptídicos y mayores grados de tolerancia a cobre ya había sido previamente reportado 9 . Es así como el gen $\operatorname{tcr} B$ perteneciente a un operón de resistencia a cobre, asociado a un plásmido conjugativo que porta genes de resistencia a VAN y ERY, fue descrito en cepas de Enterococcus spp. aisladas de cerdos ${ }^{9}$. Este operón ha sido identificado en aislados de diversas fuentes, incluyendo, en baja frecuencia, humanos sanos y ambiente hospitalario ${ }^{16}$, y se ha comprobado su capacidad de co-transferencia junto con genes de resistencia a antimicrobianos ${ }^{16}$. No obstante, la ausencia de evidencia molecular no permite comprobar la presencia de mecanismos adicionales de tolerancia a cobre iónico y se debe postular también la posibilidad de que estas diferencias en los niveles de tolerancia al metal se deban a distintos niveles de expresión de los sistemas intrínsecos de expulsión de cobre de las cepas resistentes a VAN, los cuales estarían ausentes en ambas especies de cepas ESV, donde no se pudo observar diferencias significativas entre las $\mathrm{MG}_{\mathrm{CIM}}$ de $\mathrm{CuSO}_{4}$ de E. faecalis y E. faecium (2,88 y $2.68 \mathrm{mM}$, respectivamente).

En el caso de las cepas de $S$. aureus, la situación es distinta a la detectada en Enterococcus spp., ya que las diferencias entre los grados de tolerancia a cobre obser-

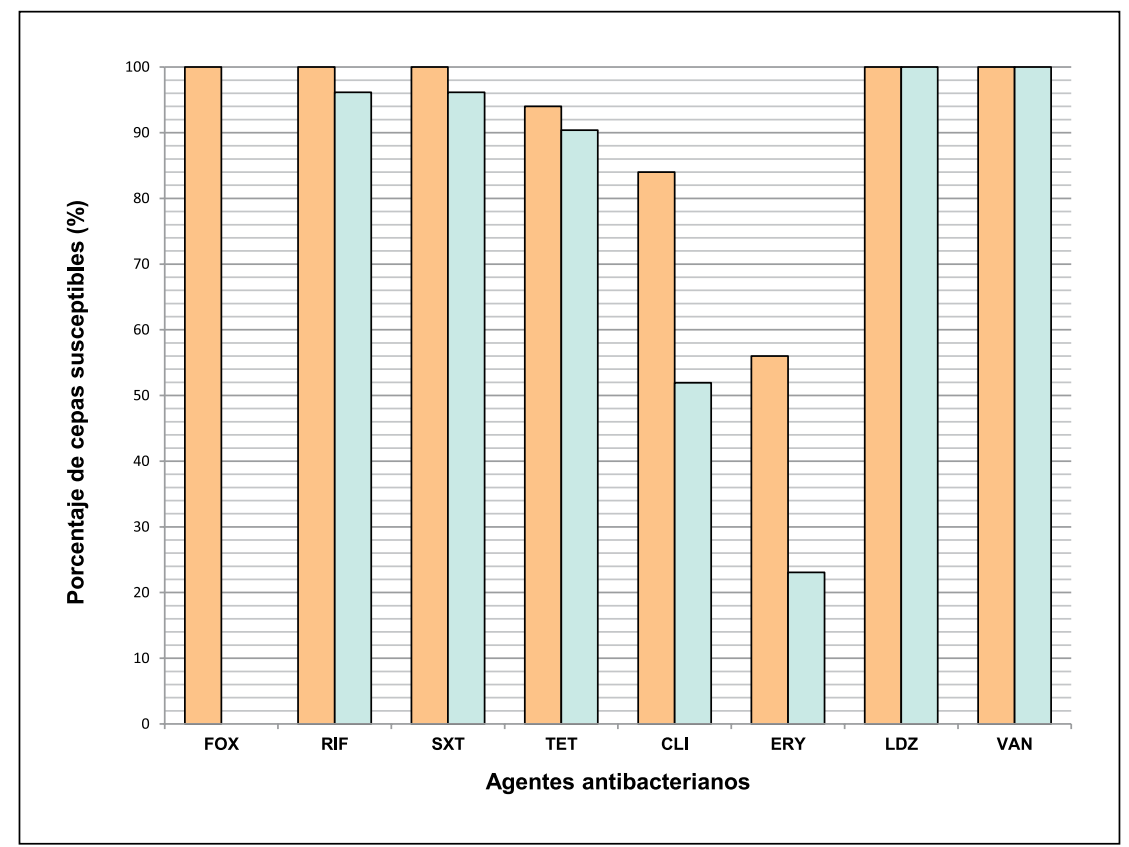

Figura 2. Actividad de agentes antibacterianos de relevancia clínica sobre cepas de $S$. aureus sensibles (barras naranja) y resistentes (barras celeste) a meticilina. FOX: cefoxitina, RIF: rifampicina, SXT: cotrimoxazol, TET: tetraciclina, CLI: clindamicina, ERY: eritromicina, LZD: linezolid, VAN: vancomicina.

vado entre las cepas SARM y SASM no son estadísticamente significativas. Aunque, la mayor $\mathrm{MG}_{\mathrm{CMI}}$ de $\mathrm{CuSO}_{4}$ observada en las cepas de SARM podría ser signo de una tendencia en el comportamiento de esta especie frente a la presencia de cobre. En un estudio realizado en Inglaterra ${ }^{17}$ se informa que al implementar el uso de aleaciones de cobre en diversas superficies de contacto, se observó una disminución de los recuentos totales de bacterias en estas superficies; sin embargo, hubo una mayor disminución de los recuentos para las cepas de SASM en relación a sus contrapartes resistentes a meticilina. Este fenómeno podría explicarse por la presencia adicional de genes de tolerancia a cobre como el operón copBmco, descrito tanto 
en asociación a plásmidos como inserto en el cromosoma bacteriano, el cual confiere mayores grados de tolerancia a cobre iónico y además ha sido transferido entre cepas de $S$. aureus $^{18}$. Asimismo, se ha descrito la presencia del gen $\operatorname{copB}$ asociado al cassette cromosomal SCCmec de SARM $^{19}$.

Los resultados obtenidos en este estudio son concordantes con lo descrito en un estudio anterior ${ }^{13}$, donde se evaluó la relación de tolerancia a cobre iónico con la resistencia a antimicrobianos en bacilos gramnegativos multi-resistentes, observándose esta asociación de modo especie dependiente ${ }^{13}$. El fenómeno presente en las cepas ERV pone en evidencia una preocupante situación con relación al tratamiento y la potencial selección de estos fenotipos en ambientes hospitalarios donde se utilice cobre como agente antimicrobiano de superficies. Esto se debe a que se ha descrito que la presencia de mayores grados de tolerancia a cobre iónico otorgaría una mayor sobrevida bacteriana en superficies cobrizadas ${ }^{20,21}$, lo que se explica debido a que la actividad antimicrobiana del metal es mediada por la liberación de iones de cobre ${ }^{12}$. Cabe señalar que estos sistemas permiten contrarrestar ciertos rangos de concentraciones de cobre iónico; sin embargo, una exposición prolongada a superficies cobrizadas provocaría una acumulación de estos iones produciendo un daño celular irreversible. Es por ello que esto no apunta a una persistencia bacteriana indefinida en estas superficies, sino que a aumentos en la sobrevida que podría llevar a la selección de cepas multi-resistentes; para lo cual sería sumamente relevante realizar a futuro ensayos de supervivencia de estas cepas en superficies de cobre. Todo esto favorecerá un uso seguro de las superficies de cobre como bactericidas, de manera de mantener su efectividad a largo plazo y evitar el riesgo de propagación de cepas multi-resistentes a los antimicrobianos, lo que puede acrecentar aún más la delicada situación epidemiológica de la resistencia bacteriana a los antimicrobianos en los hospitales.

Agradecimientos. A la Facultad de Ciencias Biológicas de la Universidad de Concepción por el financiamiento entregado para la realización de este estudio. A los Microbiólogos del Hospital "Dr. Guillermo Grant B.” de Concepción y al Instituto de Salud Pública de Chile, que proporcionaron las cepas incluidas en este estudio.

\section{Resumen}

Introducción: Las infecciones nosocomiales, producidas por bacterias cocáceas grampositivas multi-resistentes constituyen un serio problema para los sistemas de salud pública mundial. El uso de superficies de cobre en ambientes hospitalarios ha demostrado ser una alternativa efectiva para el control de diversos microorganismos, incluyendo patógenos nosocomiales multi-resistentes. Objetivo: Determinar la asociación entre la multi-resistencia a antimicrobianos y mayores niveles de tolerancia a cobre en bacterias cocáceas grampositivas aisladas de hospitales chilenos, que podrían conferir una ventaja selectiva en ambientes cobrizados. Material y Métodos: Se evaluó los niveles de tolerancia a cobre iónico en grupos de cepas de Staphylococcus aureus y Enterococcus spp. con distintos grados de susceptibilidad a antimicrobianos de relevancia clínica, mediante el método de dilución en agar Mueller Hinton. Resultados: Se observó una asociación estadísticamente significativa entre mayores niveles de tolerancia a cobre iónico y la multi-resistencia a antimicrobianos en Enterococcus spp.

\section{Referencias bibliográficas}

1.- López-Pueyo M, Barcenilla-Gaite F, AmayaVillar R, Garnacho-Montero J. Multirresistencia antibiótica en unidades de críticos. Med Intensiva 2011; 35: 41-53.

2.- MINSAL. Ministerio de Salud de Chile. 2012. Informe de Vigilancia de Infecciones Asociadas a la Atención en Salud, Ministerio de Salud de Chile. Disponible en: http://web.minsal.cl/sites/ default/files/files/informeinfeccionesChile2012. pdf (Consultado: 08 de diciembre de 2015).

3.- Fisher K, Phillips C. The ecology, epidemiology and virulence of Enterococcus. Microbiol 2009; 155: $1749-57$

4.- Rivera A, Boucher H. Current concepts in antimicrobial therapy against select Grampositive organisms: methicillin-resistant Staphylococcus aureus, penicillin-resistant pneumococci, and vancomycin-resistant enterococci. Mayo Clin Proc 2011; 86: 1230-43.

5.- Weber D, Rutala W. Self-disinfecting surfaces review of current methodologies and future prospects. Am J Infect Control 2013; 41: S31-S35.

6.- Elguindi J, Hao X, Lin Y, Alwathnani H, Weim $\mathrm{G}$, Rensing C. Advantages and challenges of increased antimicrobial copper use and copper mining. Appl Microbiol Biotechnol 2011; 91: 237-49.

7.- $\quad$ Grass G, Rensing C, Solioz M. Metallic copper as an antimicrobial surface. Appl Environ Microbiol 2011; 77: 1541-7.

8.- O'Gorman J, Humphreys H. Application of copper to prevent and control infection. Where are we now? J Hosp Infect 2012; 81: 217-23.

9.- Hasman H, Aarestrup F. $\operatorname{tcr} B$, a gene conferring transferable copper resistance in Enterococcus faecium: occurrence, transferability, and linkage to macrolide and glycopeptide resistance. Antimicrob Agents Chemother 2002; 46: 1410-6.

10.- Perron K, Caille O, Rossier C, Van Delden C, Dumas J, Köhler T. CzcR-CzcS, a twocomponent system involved in heavy metal and carbapenem resistance in Pseudomonas aeruginosa. J Biol Chem 2004; 279: 8761-8.

11.- Sandegren L, Linkevicius M, Lytsy B, Melhus A, Andersson D. Transfer of an Escherichia coli ST131 multiresistance cassette has created a Klebsiella pneumoniae-specific plasmid associated with a major nosocomial outbreak. J Antimicrob Chemother 2012; 67: 74-83.

12.- Espírito Santo C, Taudte N, Nies D, Grass G. Contribution of copper ion resistance to survival of Escherichia coli on metallic copper surfaces. Appl Environ Microbiol 2008; 74 : 977-86. 
13.- Kappes T, Domínguez M, Bello H, Mella S, Riedel G, González-Rocha G. Actividad de cobre sobre bacilos gramnegativos multirresistentes aislados en hospitales chilenos. Rev Chilena Infectol 2012; 29: 622-7.

14.- Clinical and Laboratory Standards Institute. Performance Standards for Antimicrobial Disk Susceptibility Tests; Approved StandardEleventh Edition. CLSI document M02-A11. Clinical and Laboratory Standards Institute, Wayne, Pennsylvania, USA, 2013.

15.- Clinical and Laboratory Standards Institute. Methods for Dilution Antimicrobial Susceptibility Tests for Bacteria That Grow Aerobically; Approved Standard-Ninth Edition. CLSI document M07-A9. (ISBN 1-56238-783-
9). Clinical and Laboratory Standards Institute, Wayne, Pennsylvania, USA, 2013.

16.- Silveira E, Freitas A, Antunes P, Barros M, Campos J, Coque T, et al. Co-transfer of resistance to high concentrations of copper and first-line antibiotics among Enterococcus from different origins (humans, animals, the environment and foods) and clonal lineages. $\mathrm{J}$ Antimicrob Chemother 2014; 69: 899-906.

17.- Karpanen T, Casey A, Lambert P, Cookson B, Nightingale P, Miruskenko L, et al. Antimicrobial efficacy of copper alloy furnishing in the clinical environment: a crossover study. Infect Control Hosp Epidemiol 2012; 33: 3-9.

18.- Baker J, Sengupta M, Jayaswal R, Morrissey J. The Staphylococcus aureus CsoR regulates both chromosomal and plasmid-encoded copper resistance mechanisms. Environ Microbiol 2011; 13: 2495-507.

19.- Li S, Skov R, Han X, Larsen A, Larsen J, Sørum M, et al. Novel types of staphylococcal cassette chromosome mec elements identified in clonal complex 398 methicillin-resistant Staphylococcus aureus strains. Antimicrob Agents Chemother 2011; 55: 3046-50.

20.- Elguindi J, Wagner J, Rensing C. Genes involved in copper resistance influence survival of Pseudomonas aeruginosa on copper surfaces. J Appl Microbiol 2009; 106: 1448-55.

21.- Molteni C, Abicht H, Solioz M. Killing of bacteria by copper surfaces involves dissolved copper. Appl Environ Microbiol 2010; 76 : 4099-101. 